INVESTIGACIÓN

\title{
ESTRATEGIA PARA EL CONTROL DE CONTAMINACIÓN DE FLUIDOS EN EL TREN DE POTENCIA DE EQUIPOS MINEROS
}

\section{STRATEGY FOR THE CONTROL OF FLUID CONTAMINATION IN THE POWER TRAIN OF MINING EQUIPMENT}

\author{
MSc. Edwin Espinel Blanco ${ }^{a}$, MSc. Eder Florez Solano b, MSc. Gustavo Guerrero Gómez c \\ ${ }^{a}$ Universidad Francisco de Paula Santander Ocaña, Grupo de investigación GITYD \\ Calle 9No 37-01 Apto 302, Ocaña, Colombia, eeespinelb@ufpso.edu.co \\ ${ }^{\mathrm{b}}$ Universidad Francisco de Paula Santander Ocaña, Grupo de investigación INGAP \\ Calle 8\# 3-15, Ocaña, Colombia, enflorezs@ufpso.edu.co \\ 'Universidad Francisco de Paula Santander Ocaña, Grupo de investigación GITYD \\ Calle 19 \# 7-102, Ocaña, Colombia, gguerrerog@ufpso.edu.co
}

Fecha de recepción: 12-09-2016

Fecha de aprobación: 05-12-2016

\begin{abstract}
Resumen: La inadecuada gestión de mantenimiento, es la principal causa de baja disponibilidad vehicular y altos costos originados por consumos de repuestos, fallas y paradas innecesarias para una empresa Los contaminantes en los Fluidos de un sistema de transmisión de potencia son la principal causa del mal funcionamiento y desgaste de la maquinaria, el control efectivo de la contaminación es la principal estrategia cuando se trata de implementar la filosofía del Mantenimiento Proactivo (Controlar y eliminar la causa de la falla).

En la Empresa Carbones de la Jagua (CDJ) dedicada a la extracción de carbón a cielo abierto, se presentaban continuas fallas en los equipos mineros durante el periodo de garantía ocasionados por la degradación de los lubricantes y la contaminación del combustible, lo que obligaba al proveedor GECOLSA a responder por los daños en las máquinas, los cuales consumían grandes cantidades de recursos (mano de obra, repuestos, insumos), esto obligo a GECOLSA a diseñar unas estrategias que les permitió controlar los niveles de contaminación en los fluidos (en este caso las partículas microscópicas presentes en los aceites, grasas y combustible) para disminuir las fallas durante el periodo de garantía de los componentes. Se estableció el plan de control de contaminantes, que consiste en aplicación de tareas proactivas de limpieza e inspección y en una estandarización de los procedimientos para realizar dichas actividades y los trabajos de mantenimientos programados, además se aplicaron los principios del mantenimiento productivo para organizar el taller y el almacén de repuestos.
\end{abstract}


Palabras clave: Contaminación, aceites, Mantenimiento y Fallas.

Abstract: Contaminants in the Fluids of a power transmission system are the main cause of malfunction and wear of machinery, effective control of pollution is the main strategy when it comes to implementing the philosophy of Proactive Maintenance (Control and eliminate the Cause of the fault).

At the Carbones de la Jagua Company (CDJ) dedicated to the extraction of open pit coal, there were continuous failures in the mining equipment during the warranty period caused by the degradation of the lubricants and the contamination of the fuel, which forced the GECOLSA to respond for damages in the machines, which consumed large amounts of resources (labor, spare parts, supplies), this obliged GECOLSA to design strategies that allowed them to control the levels of contamination in the fluids (in this If microscopic particles present in oils, grease and fuel) to reduce faults during the warranty period of the components. The pollutant control plan was established, consisting of the application of proactive cleaning and inspection tasks and a standardization of the procedures to carry out these activities and the scheduled maintenance work. In addition, the principles of productive maintenance were applied to organize the workshop and the spare parts store.

Keywords: Pollution, Oils, Maintenance and Failures.

\section{INTRODUCCIÓN}

En la empresa minera carbones de la jagua (CDJ) se presentó un serio problema de contaminación del aceite en los sistemas mecánicos de las máquinas, esto sucedió aun en los equipos nuevos que estaban en periodo de garantía, lo cual fue un verdadero inconveniente para el proveedor, porque lo obligaba a incrementar sus gastos por cubrimiento de garantías. Por esta razón se proyectó diseñar y aplicar un programa de control de agentes contaminantes para mejorar el consumo de recursos disminuyendo la ocurrencia de las fallas y de las paradas innecesarias.

El control de la contaminación ofrece rentabilidad a cualquier empresa minera, los pequeños detalles pueden hacer una gran diferencia, al reducir los agentes externos en los fluidos de las máquinas, en este caso las partículas microscópicas presentes en los aceites, ocasionados por la gran cantidad de material particulado presente en el ambiente, la contaminación en sistemas hidráulicos y de lubricación pueden ser de naturaleza variada que causan un impacto severo en el uso del componente y conducen a la fatiga prematura (Bensch, 2010).

El control de la contaminación se inicia con una comprensión profunda de los daños que puede causar y por qué es importante mantener los contaminantes fuera de su equipo. Básicamente, la contaminación es cualquier material no deseado en un aceite, que, si está presente puede llegar a ser una causa importante de falla para el equipo (Charles Boswell, 2003).

Los principales agentes causantes de la contaminación en una mina de extracción de carbón a cielo abierto son: losdesechos sólidos, el agua, el flujo de calor, el material particulado y el aire, los cuales entran a los aceites del equipo a través de los respiraderos, lubricadores y los sellos 
durante su operación normal y en las jornadas de mantenimiento.

Tal y como lo establece en su trabajo (Ramirez, 2007), la aplicación adecuada de estrategias para eliminar la contaminación optimiza el uso de los recursos y aumenta la efectividad de las acciones de mantenimiento incrementado la disponibilidad del equipo.

El impacto de un exhaustivo programa de control de contaminación, en combinación con la capacitación de los operadores y técnicos mecánicos logra extender la vida útil de los principales componentes de los equipos mineros ahorrándole millones a la mina y alargando la vida de los equipos, así mismo reduce los tiempos intermedios para las tareas de mantenimiento, las cuales no se prolongan demasiado, en otras palabras se presenta reducción de trabajos programados y se obtienen mejora en la disponibilidad del equipo o flota aumentado su eficiencia global de operación (Aguado, 2010) .

Como lo manifiesta (Barnes, 2010), un programa de control debe concentrarse en mantener los contaminantes fuera de la maquinaria y al lubricante en buenas condiciones de salud, a través de la aplicación de estrategias que permitan controlar los focos de contaminación, mediante la estandarización de los procedimientos y la capacitación, entrenamiento y motivación del personal.

En su trabajo (Arnold, 2009) hace claridad de la importancia, que un plan de control de contaminación este soportado por una programación de análisis de aceites y de frecuencias de muestreo, como instrumento válido para comprobar la efectividad de las actividades realizadas para disminuir la presencia de elementos exógenos en los aceites, así mismo, (Altmann, 2005) en su presentación muestra las pautas a considerar para hacer los análisis a las muestras y su beneficio como herramienta en la toma de decisiones.

Con base en estas consideraciones se implementó un programa que consistía en detectar cuáles eran las principales razones que generaban la contaminación de los aceites en la mina, para proceder a controlarlas organizando los sitios de trabajo, estandarizando procedimientos y capacitando al personal. Para verificar la efectividad del programa se realizaron análisis a muestras de aceite tomadas cada 2000 horas de servicio, en las cuales se determinaban los niveles de arena (Presencia de $\mathrm{Si}$ ) y el desgaste de los componentes (presencia de Fe).

\section{METODOLOGÍA}

Se desarrolló un programa para el control de la contaminación en el aceite del tren de potencia de los equipos mineros, inicialmente de identificaron los problemas causantes de la contaminación para posteriormente implementar estrategias enfocadas a su eliminación. Finalmente se tomaron muestras de aceites que fueron llevadas al laboratorio y se encontró disminución en la cantidad de agentes contaminantes.

\section{Identificación de Problemas Causantes de la Contaminación de los Aceites de los Equipos en la Mina Carbones de la Jagua (CDJ).}

La empresa Carbones de la Jagua CDJ, contrata con GECOLSA el suministro de sus equipos mineros y el mantenimiento de los mismos durante el periodo de garantía. En la mina CDJ, el proveedor (GECOLSA), se encontró con que los equipos mineros presentaban continuas fallas durante el 
periodo de garantía que causaban paradas repetitivas que afectaban la producción y aumentaban los costos por mantenimiento y reposiciones por componentes averiados.

Estas fallas en su mayoría eran producto de la acumulación de agentes contaminantes en los equipos mineros, las cuales ocasionaban retrasos en la ejecución de los trabajos de mantenimiento preventivo (PM), por lo cual los trabajos no se realizaban en el tiempo programado, haciendo necesario una reprogramación, que obligaba a los equipos a estar por mayor tiempo indisponibles, disminuyendo la producción y aumentado los costos operacionales de la mina.

Al sistema del equipo minero entraban contaminantes que afectaban su correcto funcionamiento, la mayoría de estos problemas se presentaba en componentes que todavía estaban en periodo de garantía.

Estos inconvenientes generaron que los trabajos de mantenimiento se retrasaran y los tiempos entre fallas disminuyeran, generando costos adicionales. Se propuso identificar las principales causas que ocasionaban la contaminación de los fluidos en los sistemas de los equipos. Como primera medida realizó un diagnóstico respecto a la efectividad de los trabajos y los procedimientos.

Se llevo a cabo una auditoría a las instalaciones del taller de mantenimiento, a la sección de lavado de los equipos, al almacén y a la súper carpa (bodega para almacenar componentes grandes), proceso que consistió en hacer un registro fotográfico de los espacios, los equipos y los componentes, así mismo, en entrevistar a los técnicos de mantenimiento, para detectar cuáles eran los problemas más comunes y por qué se presentaban.

En la auditoría se verificó el estado del piso o terreno, los bancos de trabajo, las bahías de Mantenimiento Preventivo, la efectividad de los procesos de suministro y drenaje de aceites, la remoción e instalación de componentes, el estado de protección de los sistemas de las máquinas, la condición de los elementos para tomar las muestras de aceite, el estado de las herramientas y accesorios, así como los procedimientos para la disposición de residuos peligrosos.

La información recolectada durante el proceso de auditoría fue analizada y se detectaron problemas para controlar los contaminantes en: el lavado de los equipos, las condiciones del piso y la ubicación del taller, la manipulación de los componentes o piezas y los procedimientos para el suministro y drenado de aceites.

Lavado de los equipos.Las instalaciones del control de lavado no eran las mejores. El sistema de lavado que se utilizaba no brindaba el adecuado flujo volumétrico de agua a la presión requerida, para limpiar la máquina o componente de una manera eficaz, además cuando los trabajos se retrasaban los equipos no eran lavados antes de ser llevados al taller. En términos generales el equipo estaba llegando a la sección de mantenimiento con demasiado barro, en la figura 1, se puede observar las condiciones en las que estaban llegando los equipos a la sección de mantenimiento para ser intervenidos.

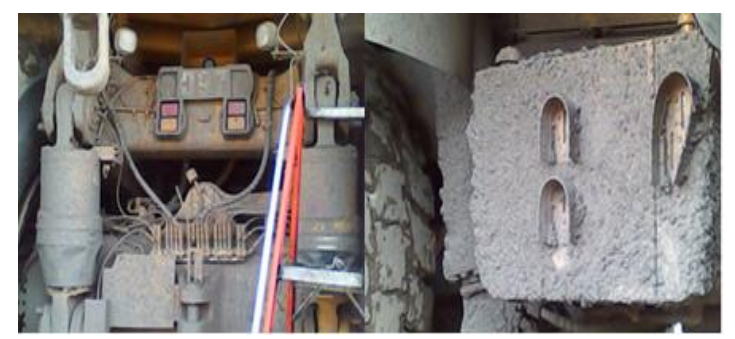

Figura 1. Equipo sin el lavado adecuado para mantenimiento preventivo

Fuente: Elaboración propia

Las condiciones del piso. El taller estaba ubicado en una zona de tráfico y su piso no 
cumplía con las condiciones adecuadas que se recomienda para un control de contaminación. Este era un sitio abierto, sin una estructura que protegiera tanto al equipo como al técnico de la lluvia, la instalación no contaba con un piso en concreto o asfalto, de manera que, cuando se estaban realizando alguna intervenciones a los equipo y se presentaban lluvias repentinas, entraba al sistema gran cantidad de agua y de partículas sólidas.

Otro problema detectado es, que el piso se encuentra desnivelado y al momento de realizar trabajos específicos como una calibración de suspensión, no se lograba verificar la ejecución correcta de los mismos. En la figura 2, se puede apreciar la necesidad de acordonar la zona, dada ubicación del taller en un sector de tráfico, así mismo se evidencias las inadecuadas condiciones del piso y la carencia de un techo o cubierta.

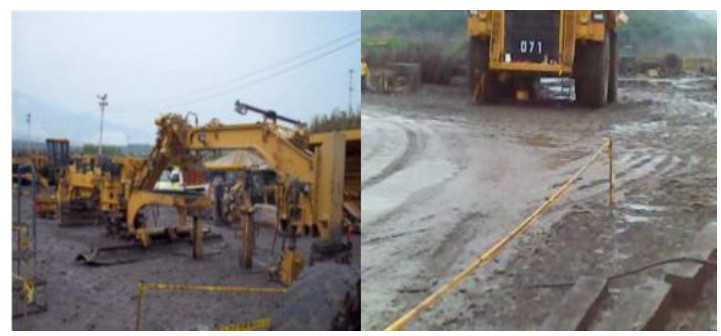

Figura 2. Condiciones del piso y ubicación del taller. Fuente: Elaboración propia

\section{La manipulación de los componentes o}

piezas. Tanto en el almacén como en taller no se le estaban dando un adecuado manejo a los componentes. En el almacén no se conservaban algunos repuestos en su empaque original $o$ en las condiciones recomendadas, se apilaban los repuestos unos sobre otros sin los cuidados requeridos como se puede apreciar en la figura 3, así mismo, la ejecución de los trabajos en el taller no se estaban realizando con el debido cuidado de las piezas de forma que se lograban contaminar el componente y el sistema, pues las piezas se dejan sobre la tierra del piso del taller y no se protegen de agentes externos como el agua y el polvo (figura 4).

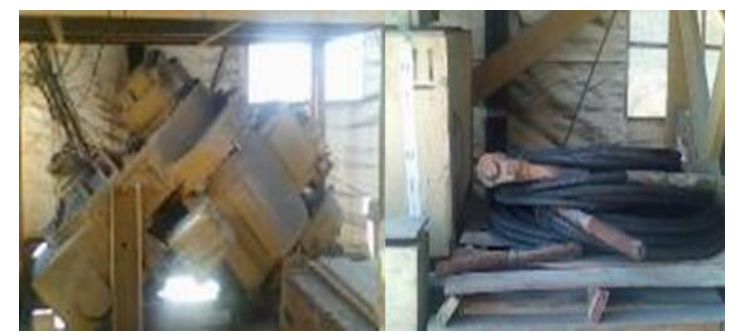

Figura 3. Repuesto inadecuadamente organizados en el almacén. Fuente: Elaboración propia

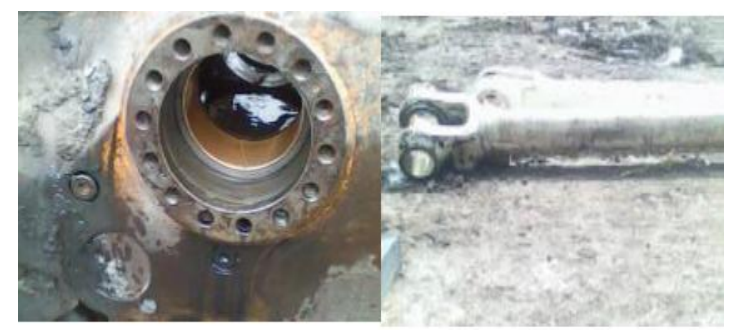

Figura 4. Componentes no protegidos durante la ejecución de trabajos de mantenimiento Fuente: Elaboración propia

Procedimientos para el suministro y drenado de aceites. Se detectó que en general los procedimientos para el suministro y drenado de aceite a los equipos no estaban bien estructurados $y$ no contemplaban los cuidados necesarios para controlar los niveles de contaminación en los sistemas de los equipos mineros. En general, los trabajos no se realizaban con la mayor precaución para evitar la entrada de contaminantes, pues se hacían utilizando equipos portátiles donde no se protegían las líneas de suministro, tal y como se observa en la figura 5, motivo por el cual, ingresaban partículas que deterioraban los componentes. Otra situación encontrada era la falta de estándares de limpieza y mantenimiento programado para el cambio de respiraderos, filtros y líneas del sistema de abastecimiento, elementos que en mal estado permitían el acceso de partículas al 
lubricante o en algunos casos eran los generadores de la contaminación.

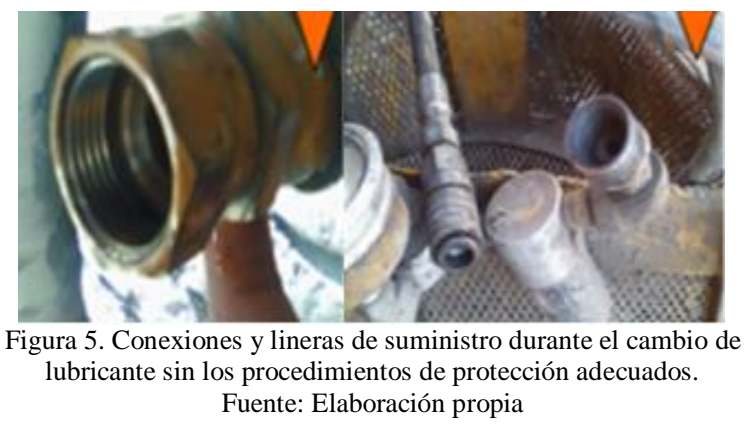

A través de la auditoria se pudo constatar que los gastos por reposiciones de componentes por concepto de garantías y servicios de mantenimiento, eran ocasionados por el alto grado de contaminación en los lubricantes, lo que deterioraba los sistemas pertenecientes al tren de potencia.

Estas circunstancias obligaron diseñar unas estrategias que pudieran ser implementadas $\mathrm{y}$ permitieran disminuir la presencia de elementos contaminantes en los lubricantes.

\section{Implementación de Estrategia para el Control de Contaminantes.}

Conociendo las principales razones causantes de la contaminación de los lubricantes pertenecientes al tren de potencia de los equipos en la empresa CDJ, se diseñó y se procedió a implementar un programa de control de contaminantes que involucraba una serie de estrategias organizadas fundamentadas en las condiciones y el contexto operacional de los equipos en la mina, aspectos que permitieran determinar las tareas proactivas que debían ejecutarse para garantizar el funcionamiento de los componentes y sistemas (Moubray, 2004).

Estas estrategias se diseñaron considerando principios del Mantenimiento Productivo Total TPM, como lo es el trabajo en equipo, la capacitación del personal, la motivación y reconocimiento, el mantenimiento autónomo y la estandarización de procedimientos, para buscar el cero defectos, cero averías y cero desperdicios (Nakajima, 1991).

De esta forma la implementación del programa de control de contaminantes a los aceites del tren de potencia de los equipos en la mina CDJ, consistía en realizar las siguientes actividades:

- Estandarización de procedimientos para conservar limpios y ordenados los equipos y las áreas de trabajo.

- Capacitaciones y charlas de motivación a los técnicos, mecánicos y operarios para controlar la contaminación en la mina.

- Mejoras a los procedimientos para ejecutar las actividades de mantenimiento.

- Organización del almacén de repuestos para garantizar el correcto almacenamiento de los componentes y equipos.

- Adecuación de las condiciones del taller de mantenimiento para ejecutar correctamente los trabajos.

- Auditoria a las actividades de mantenimiento realizadas a los equipos.

\section{La Estandarización de procedimientos para} conservar limpios y ordenados los equipos y las áreas de trabajo. Se realizó una limpieza general a las áreas de trabajo y a los equipos mineros que permitió eliminar gran cantidad de elementos que eran fuente de contaminación, como piezas inservibles, desechos, acumulación de basura y desperdicios de materiales e insumos. 
Seguidamente se procedió a programar y estandarizar las tareas básicas de mantenimiento relacionadas con la limpieza, lubricación e inspección que debían realizárseles a los equipos y las áreas de trabajo para mantenerlos en condiciones libres de contaminantes.

Capacitaciones y charlas de motivación a los técnicos, mecánicos y operarios para controlar la contaminación en la mina. Se realizaron jornadas de capacitación a los técnicos, mecánicos y operarios de los equipos para mostrarles las ventajas de mantener su sitio de trabajo limpio y ordenado, e igualmente los alcances de cumplir con las actividades básicas de mantenimiento autónomo y los beneficios de mantener sus sitios de trabajo y las herramientas organizadas y limpias. Finalmente se estructuro y se les dio a conocer un plan de incentivos que podrían obtener por disminuir los niveles de contaminación en los equipos y por ende la presencia de fallas catastróficas.

\section{Mejoras a los procedimientos para ejecutar} las actividades de mantenimiento. Se reestructuraron los procedimientos de los trabajos de mantenimiento programado para eliminar la presencia de contaminantes durante la ejecución de los mismos, de esta forma, se incorporó el lavado general de los equipos y componentes antes de ingresar a mantenimiento, en la figura 6 se puede apreciar que los equipos se comenzaron a lavar adecuadamente antes de ingresar al taller lo cual disminuyo la posibilidad de que los lubricantes se contaminen al momento de ejecución de los trabajos.

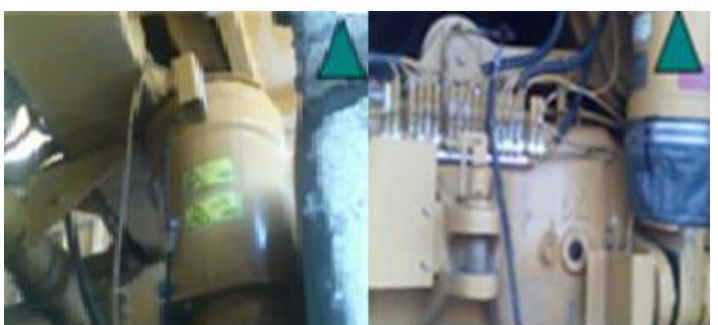

Figura 6. Equipos correctamente lavados al momento de ingresar al taller.

Fuente: Elaboración propia

De la misma forma se elaboraron instructivos de procedimiento que cumplen con la normativa dispuesta por el proveedor de los equipos para su intervención, igualmente se dispusieron normas y procedimientos para el cambio y disposición final de los fluidos.

\section{Organización del almacén de repuestos} para garantizar el correcto almacenamiento de los componentes y equipos. Se organizó el almacén de repuestos de acuerdo a la frecuencia de uso y requerimientos de almacenamiento de las piezas, componentes y equipos. En esta etapa también se elaboraron instructivos para la disposición de los de los elementos en el almacén con las consideraciones necesarias para garantizar su operatividad en el momento cuando se han requeridos.

En la figura 7, se puede apreciar el estado del almacén de repuestos después de la limpieza y organización general, así como la disposición de los repuestos de acuerdo a las normas establecidas por el fabricante que fueron incluidas en los estándares de procedimientos definidos para el almacenamiento de repuestos.

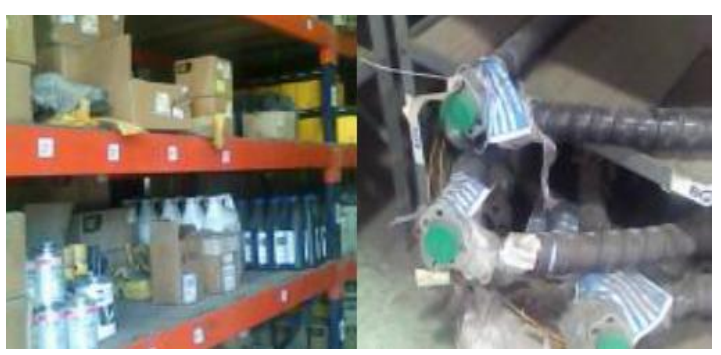


Figura 7. Repuestos organizados en el almacén de acuerdo a las recomendaciones.

Fuente: Elaboración propia

Adecuación de las condiciones del taller de
mantenimiento $\quad$ para
cojecutar mantenimiento se encontraba en condiciones inadecuadas para realizar los trabajos, el piso no tenía recubrimiento o planchón en cemento, era solamente una superficie en tierra por lo cual las piezas y repuestos se contaminaban durante la ejecución de las trabajos, por esta razón se dispuso utilizar soportes de madera para colocar en el piso durante las rutinas de mantenimiento correctivo y preventivo que evitaran que las partes y los insumos entraran en contacto con el terreno. Por la inexistencia en el taller de un techo que protegiera a los equipos que estaban en mantenimiento de factores externos como el agua y el polvo, se dispuso que durante las intervenciones se acordonara la zona y se cubrieran a los equipos y componentes para evitar la entrada de dichos a gentes a los sistemas mecánicos.

En la figura 8, se pueden apreciar algunos componentes con los cuidados necesarios para evitar su contaminación durante la ejecución de los trabajos de mantenimiento.

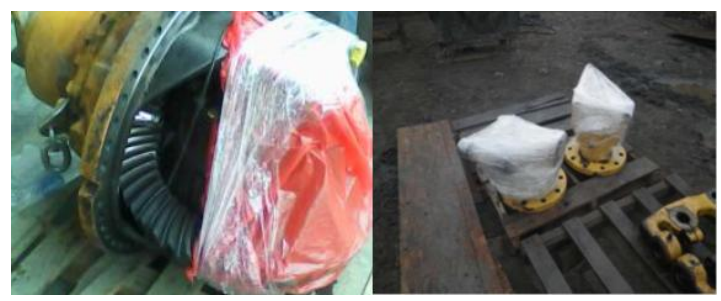

Figura 8. Componentes con los debidos cuidados para evitar la contaminación en el taller durante los trabajos de mantenimiento Fuente: Elaboración propia

\section{Auditoria a las actividades de} mantenimiento realizadas a los equipos. Se diseñó un formato de auditorías y supervisión para hacer seguimiento a los trabajos de mantenimiento y a las instalaciones del taller y almacén. Los formatos de registro para controlar los niveles de contaminación en la mina fueron incorporados al sistema de información de mantenimiento de la empresa CDJ para ser utilizados permanentemente.

La figura 9, muestra el formato de registro utilizado en las auditorias diarias para controlar las actividades de mantenimiento y verificar las condiciones del almacén de repuestos y el taller.

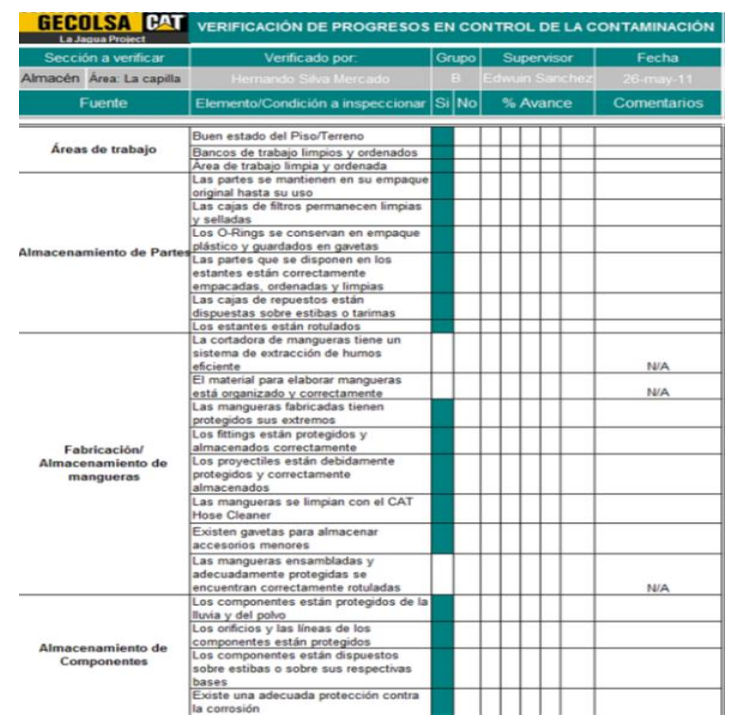

Figura 9. Formato de registro para realizar auditorías a los trabajos de mantenimiento y a las instalaciones del taller y el almacén de repuestos.

Fuente: Elaboración propia

\section{RESULTADOS}

Para verificar la efectividad de las estrategias utilizadas para controlar la presencia de contaminantes en los lubricantes, la programación de los análisis contemplaba tomar muestras a los aceites cada 2000 horas de servicio para luego ser analizadas en el laboratorio de Caterpillar, donde inicialmente se verificarían las cantidades del elemento silicio ( $\mathrm{Si}$ ) porque este indica la presencia de arena (Polvo 
Ambiental) producto fundamentalmente de un mal funcionamiento de los filtros, así mismo, se analizaría la presencia de Hierro $(\mathrm{Fe})$ en las muestras, que indica un desgaste severo en los componentes por perdida de las propiedades del aceite.

Se diseñó un instructivo de procedimiento para realizar la toma de muestras de los aceites, el cual incluía los cuidados que debían tenerse presentes para no contaminarlas. La figura 10 muestra unas botellas con distintas muestras de aceite tomadas a los equipos, las cuales son etiquetadas indicando el número de serie del equipo, el respectivo horómetro al momento de la toma, así como, el tipo y la marca del aceite.

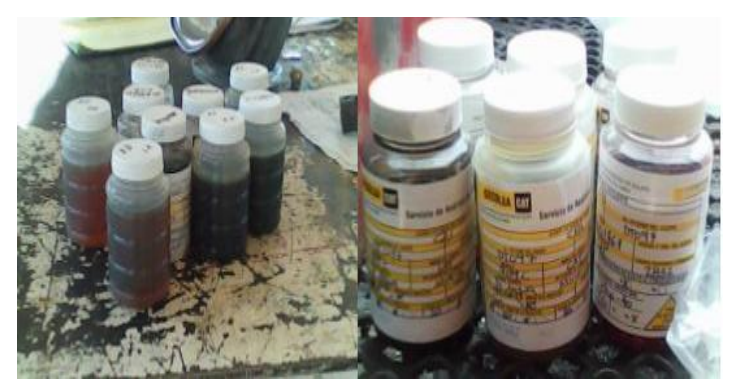

Figura 10. Muestras de aceite debidamente etiquetadas. Fuente: Elaboración propia

De esta forma se tomaron distintas muestras de los diferentes componentes para cada equipo y se analizó la presencia en cada una de ellas de elementos contaminantes. Los resultados de las pruebas de laboratorio fueron graficados por el programa para analizar tendencias (Trend Analysis Module, TAD), utilizado por Caterpillar en estos casos.

Los resultados de las pruebas de laboratorio en aproximadamente el $80 \%$ de las muestras analizadas arrojaron disminuciones en los niveles de elementos contaminantes en los lubricantes. Por esta razón en un gran número de componentes los niveles de elementos contaminantes disminuyeron notablemente, tal y como se aprecia en la figura 11 donde se muestra el pantallazo del programa TAD de la gráfica que indica la tendencia del porcentaje del elemento hierro (Fe) en el motor del equipo DT050, en la cual, se observa la disminución del elemento contaminante con cada muestra analizada.

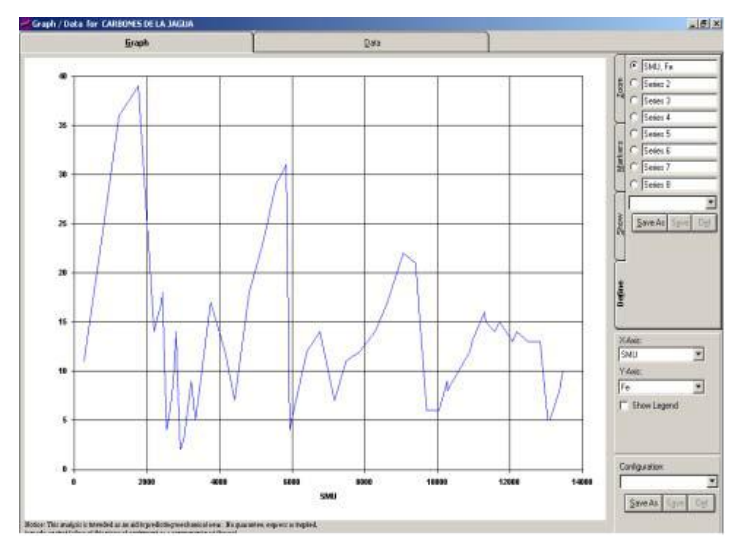

Figura 11. Porcentaje de elemento hierro en el motor del equipo DT050 cada 2000 horas de servicio. Fuente: Elaboración propia

En la figura 12, se muestra la tendencia graficada por el programa TAD, con base en los resultados de laboratorio realizados a las muestras de aceite del diferencial del equipo DT061 tomadas cada 2000 horas de servicio para determinar el porcentaje del elemento sílice ( $\mathrm{Si}$ ), en la cual se puede observar la disminución del contaminante.

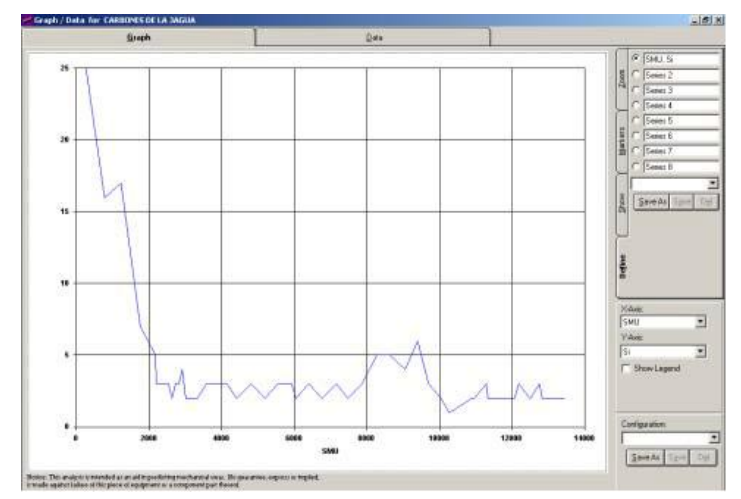

Figura 12. Porcentaje de elemento sílice en el diferencial del equipo DT061 cada 2000 horas de servicio. Fuente: Elaboración propia

El análisis de las pruebas de laboratorio arrojo que en otros componentes como en el convertidor del equipo DT083 el porcentaje 
de hierro (Fe) examinado cada 2000 horas de servicio presento una disminución en las primeras muestras pero posteriormente incrementó su presencia, tal y como se puede evidenciar en la figura 13, de la tendencia graficada por el programa TAD.

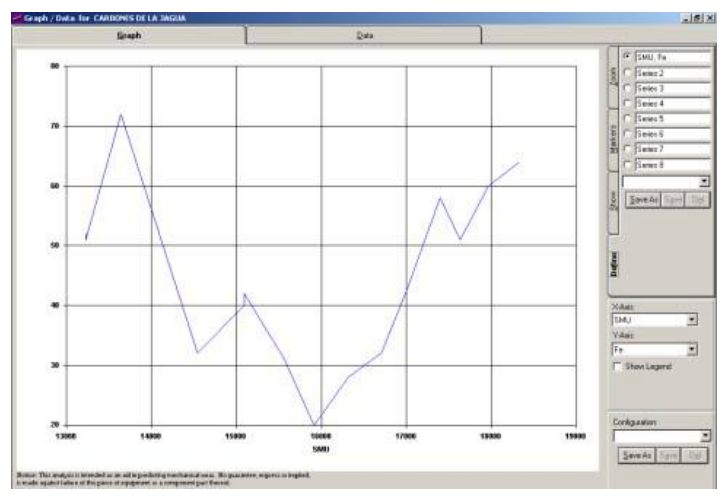

Figura 13. Porcentaje de elemento hierro en el convertidor del equipo DT083 cada 2000 horas de servicio Fuente: Elaboración propia

De la misma forma el diferencial delantero del equipo WL115 en un comienzo presento disminución en el porcentaje de contaminación del elemento sílice $(\mathrm{Si})$ que luego tendió a incrementarse, tal y como se observa en la figura 14 que muestra la tendencia graficada por el programa TAD del porcentaje del elemento sílice cada 2000 horas de servicio.

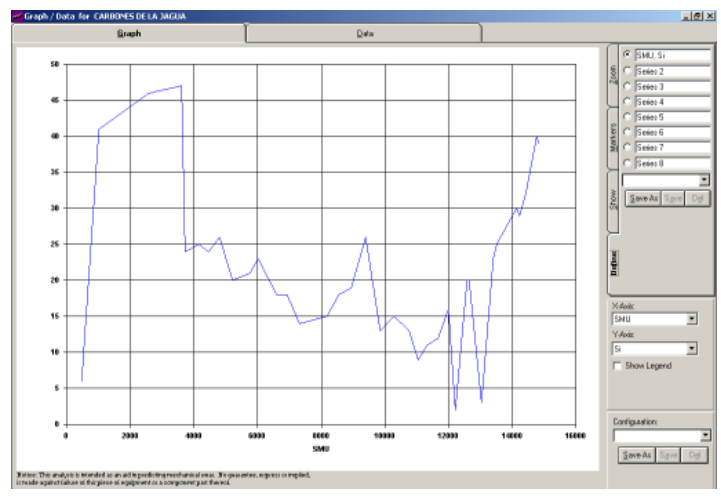

Figura 14. Porcentaje de elemento sílice en el diferencial delantero del equipo WL115 cada 2000 horas de servicio

Fuente: Elaboración propia

\section{CONCLUSIONES.}

Es de gran importancia resaltar como aspecto positivo que la implementación del programa para controlar contaminantes en la empresa CDJ, mejoro el ambiente de trabajo debido a la capacitación y motivación de los empleados por mantener la cultura del orden y limpieza, principios del mantenimiento autónomo que permitieron disminuir los tiempos en la ejecución de los trabajos.

Los resultados de los análisis realizados a las muestras de aceites tomadas de los diferentes componentes de los equipos mineros permitieron evidenciar en el $80 \%$ de las pruebas una notable tendencia a la disminución en los niveles de elementos contaminantes, lo cual permitió disminuir en el $25 \%$ las solicitudes de reposiciones por concepto de garantía.

En el $20 \%$ de las muestras los resultados de los análisis mostraron que con la aplicación de las primeras etapas del programa de control de contaminantes se pudo disminuir la presencia de elementos dañinos en los aceites, que posteriormente se incrementaron por inconvenientes al momento de la toma de las muestras como gran cantidad de material particulado en el ambiente y el uso de elementos sucios que contaminaron la cantidad de lubricante extraído para ser analizado. Esto pudo deducirse por el buen comportamiento de los sistemas que no reflejaban la cantidad de contaminantes encontrados en los resultados para los últimos periodos.

El trabajo desarrollado trajo como resultado la disminución de aproximadamente el $48 \%$ de las paradas en los equipos, ocasionadas por mal funcionamiento de los sistemas mecánicos. Así mismo, se desarrolló la cultura de la limpieza y el orden como 
principios del mantenimiento autónomo en búsqueda de la productividad total.

\section{BIBLIOGRAFÍA}

Aguado, N. (2010, septiembre 10). mantenimientoplanificado. Retrieved marzo 5, 2011, from http://mantenimientoplanificado.com

Altmann, C. (2005). El Análisis de Aceite como Herramienta del Mantenimiento Proactivo en Flotas de Maquinaria Pesada. Primer Congreso Uruguayo de Mantenimiento, Gestión de Activos y Confiabilidad. montevideo.

Arnold, P. V. (2009). Como Utilizar los Estándares de Limpieza de los Fluidos para Controlar los Costos. Machiney Lubrication.

Barnes, M. (2010). Aplique en la Lubricación un Enfoque Similar al de la Seguridad en la Planta. Machinery lubrication.

Bensch, L. (2010, junio 10). confiabilidad.net. Retrieved febrero 15, 2011, from http://www.confiabilidad.net

Charles Boswell. (2003). 6 Steps to Effectively Control Contamination. the 18th International Maintenance Conference.

Moubray, J. (2004). Mantenimiento Centrado en Confiabilidad RCM II. Argentina, arequipa: Aladon.

Nakajima, S. (1991). Mantenimiento Productivo Total. Madrid: Tecnologias de Gerencia y Produccion S.A.
Ramirez, J. F. (2007). Plan de Lubricación para el Mantenimiento mecánico para la Maquinaria Pesada Utilizada en Movimiento de Tierra, en la Industria de la Construcción, por TOPSA CONSTRUCCIONES S.A. Guatemala: Universidad de San Carlos. 